\title{
On-demand droplet loading of ultrasonic acoustic levitator and its application for protein crystallography experiments
}

\author{
Cite as: Appl. Phys. Lett. 114, 213702 (2019); doi: 10.1063/1.5095574 \\ Submitted: 11 March 2019 - Accepted: 3 May 2019 • \\ Published Online: 29 May 2019
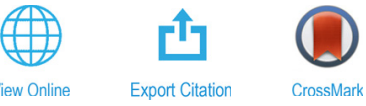

Soichiro Tsujino, ${ }^{1, a)}$ Akira Shinoda, ${ }^{1}$ and Takashi Tomizaki ${ }^{2}$

\begin{abstract}
AFFILIATIONS
'Laboratory for Micro- and Nanotechnology, Photon Science Division, Paul Scherrer Institut, CH-5232 Villigen PSI, Switzerland

${ }^{2}$ Laboratory for Macromolecules and Bioimaging, Photon Science Division, Paul Scherrer Institut, CH-5232 Villigen PSI, Switzerland

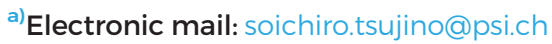

\begin{abstract}
Higher throughput has been ever demanded in the state-of-the-art protein crystallography beamlines for applications such as the screening of drug targets in protein-ligand complex structures at room temperature. As a potential method to achieve an order of magnitude higher throughput, we explore capturing of ejected droplets by an acoustic levitator to remotely load single protein crystal samples to an acoustic levitation diffractometer at the Swiss Light Source synchrotron facility. The results from X-ray diffraction experiments support the feasibility of this method as a fully automated sample delivery for high-throughput serial crystallography experiments using the acoustic levitation.
\end{abstract}

Published under license by AIP Publishing. https://doi.org/10.1063/1.5095574

Structure based drug discovery often requires multiple ligand complex structures with a structurally diverse set of compounds to investigate the interaction with the target protein in all detail. Various soaking conditions have to be also tested to increase the hit rate of the structure determinations. Such screening of compound libraries of several hundred of molecules for example by the application of Xray crystallographic methods requires substantial experimental efforts, which demand the collection of several thousand of datasets. ${ }^{1,2}$ In comparison, the typical throughput is limited to a few hundred datasets per day on the state-of-the-art protein crystallography beamlines at synchrotrons such as I04-1 of the Diamond Light Source ${ }^{3}$ due to the time consuming steps such as the preparation of sample holders and the manual harvesting of the crystals. As a method of the fast data collection from single crystals at room temperature, the use of the ultrasonic acoustic levitation was proposed. ${ }^{4}$ An acoustic levitation diffractometer (ALD) combines the ultrasonic acoustic levitation of single protein crystals in a levitated droplet and a fast-frame-rate X-ray image detector. By taking advantage of the ability to control the fast rotation of the crystal in the levitated droplet, ${ }^{5-7} \mathrm{X}$-ray diffraction images from different crystal orientations are collected at a high frame rate. ${ }^{4}$ However, without fast and efficient methods of automated crystal harvesting and delivery, it is difficult to speed up the overall throughput of $\mathrm{X}$-ray diffraction experiments based on ALD.
As shown in the literature, ${ }^{5,8}$ loading a water droplet with the radius $R$ of $0.5-1 \mathrm{~mm}$ is accomplished by simply dispensing the droplet in the levitator from a syringe. For experiments with crystals in the range of $50-200 \mu \mathrm{m}$ and smaller, it is advantageous to use droplets with $R$ below $0.1-0.3 \mathrm{~mm}$. This is for the purpose of matching the droplet size to the crystal size in order to increase the hit rate of the $\mathrm{X}$-ray beam on the sample in the levitated droplet and to maximize the signal-to-noise ratio of the diffraction intensities by minimizing the X-ray absorption and scattering by the liquid. ${ }^{9}$ Loading such small droplets becomes increasingly difficult because the acoustic radiation force is proportional to $R^{3}$ and consequently decreases rapidly with the decrease in $R$. In addition, the droplet at the tip of the capillary has to be detached by applying a force against the capillary force that is proportional to the radius $R_{c}$ of the capillary. The resulting requirement for the reduction of $R_{c}$, which needs to be proportional to $R^{2}$, is, however, not practical when we have to transfer single crystals into the droplet.

At state-of-the-art protein crystallography beamlines, sophisticated delivery methods of cryo-cooled samples using automated robotic arms have significantly increased the sample changing efficiency. ${ }^{10}$ Nevertheless, sample harvesting steps from standard crystallization plates and mounting the sample on standard sample holders remain to be time-consuming manual procedures, and mechanical crystal damage is frequently observed. Considerable improvements for 
the sample harvesting procedure have been foreseen by utilizing the acoustic radiation force for sample harvesting. ${ }^{11-14}$ Mounting protein crystal samples on standard sample holders by ejecting samples from the crystallization plates, ${ }^{15}$ as well as dispensing of microcrystals on a polymer film for serial crystallography experiments using an X-ray free electron laser by the focused acoustic radiation ${ }^{14}$ or by a drop-ondemand (DoD) dispenser, ${ }^{13}$ have been demonstrated recently. These reports indicate the potential of such droplet dispensing methods to automate the sample harvesting and delivery without damaging the sample qualities.

In this Letter, we explore a method of loading small droplets by using $\mathrm{DoD}$ dispensers to eject droplets and capturing those by the acoustic levitator. By analyzing the required condition for the trapping, we have developed a stable trapping strategy and applied the method to conduct a pilot protein crystallography experiment. In this way, we showed that the combination of the DoD dispensing method with the ALD is promising to realize an automated X-ray diffraction experiment without solid containers for crystals.

We used a single-axis acoustic levitator consisting of an ultrasonic transducer and an acoustic mirror reflector with the diameter of $20 \mathrm{~mm},{ }^{4}$ see Fig. 1. The ultrasonic transducer was driven by a boltclamped Langevin-type piezo-oscillator with the resonance frequency approximately equal to $38.5 \mathrm{kHz}$. The reflector was a concave spherical glass mirror with the focal length of $20 \mathrm{~mm}$. The horn and the mirror form an acoustic cavity. Its resonance is achieved when their separation is adjusted to approximately equal to $n$ times one half of the ultrasound wavelength ( $n$ is an integer). For the crystallography experiment, we set $n=5$ to secure a large stereo-angle from the droplet as shown in Fig. 1. The levitator cavity was adjusted to the resonance by monitoring the signal from a piezosensor attached to the mirror. The influence of droplets of $0.5-1 \mathrm{~mm}$ or smaller on the cavity resonance or on the sensor signal was absent. For dispensing droplets and protein crystal samples, we used commercial DoD dispensers that produced droplets by squeezing a polyimide capillary filled with liquid by a

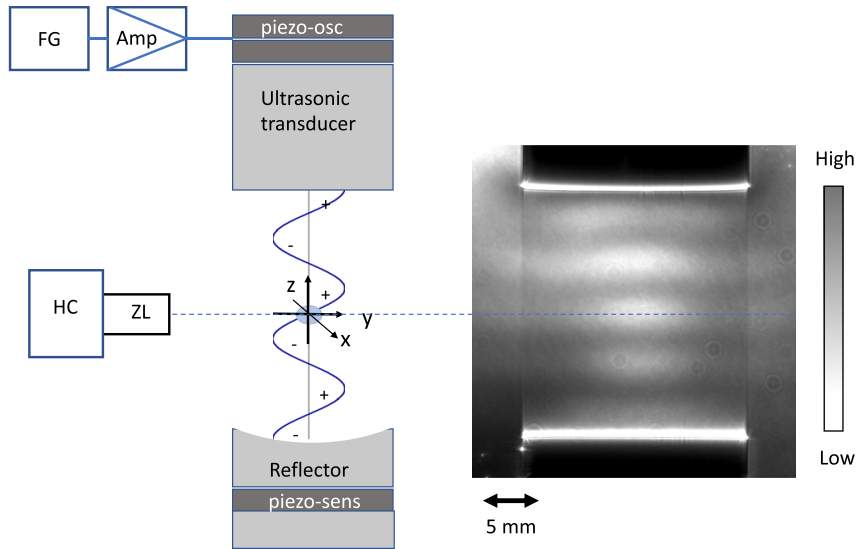

FIG. 1. Schematic of the single-axis acoustic levitator consisting of an ultrasonic transducer excited by a piezo-oscillator and a mirror reflector attached with a piezoultrasonic sensor. FG: function generator, AMP: amplifier, HC: high speed camera, and $\mathrm{ZL}:$ zoom lens. The right panel shows the time-averaged pressure distribution induced by the ultrasonic standing wave via nonlinearity of ambient air, imaged by the Schlieren method ${ }^{16,17}$ (see supplementary material Fig. S1). The bright and dark areas are the low and high pressure zones, respectively. piezodisk upon sending voltage pulses (Pipejet nano dispenser, Biofluidix $\mathrm{GmbH}){ }^{18}$ We used capillaries with the inner diameters of 0.2 and $0.5 \mathrm{~mm}$. The motion of the droplets was recorded by using a high-speed camera (Photron Mini AX100).

We applied the developed droplet dispensing method to the ALD installation on the X06SA beamline at the Swiss Light Source, Paul Scherrer Institut, ${ }^{19}$ to conduct X-ray diffraction experiments of the on-demand loaded protein crystals in a schematic setup shown in Fig. 4(a). We used lysozyme crystals with the size in the range of $100-200 \mu \mathrm{m}$ which were prepared from the lysozyme protein solution (concentration of $100 \mathrm{mg} / \mathrm{ml}$ in $50 \mathrm{mM} \mathrm{Na}$ acetate buffer at $\mathrm{pH}$ 4.5 purchased from Alfa Aeser, Wardhill, MA, USA) and mixed in the crystallization buffer consisting of 5\% polyethylene glycol (average molecular weight of 5000), $2 \mathrm{M} \mathrm{NaCl}, 50 \mathrm{mM} \mathrm{Na}$ acetate buffer at $\mathrm{pH} 4.5$, and $25 \%$ ethylene glycerol. We adjusted the ultrasound pressure of the levitator to $\sim 3.2 \mathrm{kParms}(167 \mathrm{~dB}$ at the sound pressure level, SPL, measured with respect to $20 \mu \mathrm{Pa}$ ) since we found previously that a crystal of such sizes spins a few turns per second when it is in a levitated droplet with the diameter of $0.5-1 \mathrm{~mm} .{ }^{4,19}$ The crystals were filled into the $0.5 \mathrm{~mm}$-capillary of the dispenser together with the crystallization buffer. The estimated surface tension of the crystallization buffer was smaller than that of pure water by $11 \%$. No significant change in the coalescence condition of two droplets was expected with this change. The viscosity of the crystallization buffer was estimated to be 6 times higher than that of pure water, and therefore, we increased the dispenser driving voltage by $30 \%$ to achieve the necessarily ejected droplet velocity. The X-ray beam size was adjusted to $0.4 \mathrm{~mm}$-square matching the droplet size.

First, we tested the loading of a water droplet using a single DoD dispenser. Figures 2(a)-2(c) show the selected time frames of a recorded event of the droplet loading (also see supplementary material Video 1). The droplet with an $R$ value of $\sim 0.25 \mathrm{~mm}$ was ejected from the dispenser with the $0.5 \mathrm{~mm}$-capillary and subsequently captured by the acoustic levitator. For capturing the droplet, low initial droplet velocity was necessarily. For stable operation of the DoD dispenser used here, high ejection velocities $(>0.5 \mathrm{~m} / \mathrm{s})$ were demanded. The selected ejection velocity and ejection point were the result of an
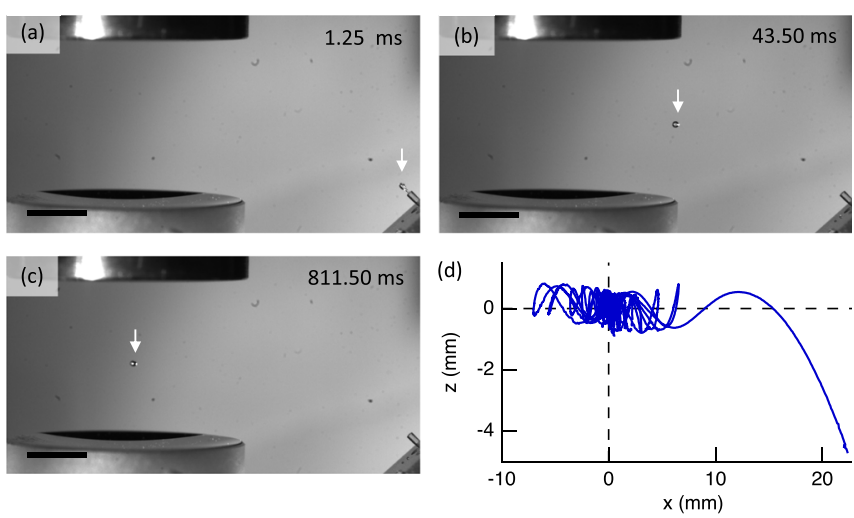

FIG. 2. (a)-(c) Selected time frames of the droplet loading to the acoustic levitator when the droplet was ejected from the drop-on-demand dispenser (right bottom corner). These were recorded using the high-speed camera at the frame rate of $4000 \mathrm{~Hz}$. The scale bar is $5 \mathrm{~mm}$. (d) shows the droplet trajectory over $1 \mathrm{~s}$ (center-of-mass motion) evaluated from the recorded event. 
optimization of these two conditions. We set the initial droplet velocity equal to $0.44 \mathrm{~m} / \mathrm{s}$ and the ejection angle equal to $45^{\circ}$ and placed the outlet of the capillary at $12 \mathrm{~mm}$ away from the levitator edge and $4.8 \mathrm{~mm}$ lower than the middle pressure node (right bottom corner). In this way, the droplet velocity at the levitator edge was reduced to $\sim 0.3$ $\mathrm{m} / \mathrm{s}$ by gravity and friction of air. Because of the difficulty in further reducing the droplet velocity, the levitator was adjusted to the 3 rd resonance instead of 5th to increase the ultrasonic pressure amplitude $P_{a c}$ to $5.0 \pm 0.2 \mathrm{kPa} \mathrm{rms}(171 \mathrm{~dB}$ in SPL), which is approximately 2.2 times larger than the $P_{a c}$ value for the crystallography experiment. In Fig. 2(d), the center-of-mass trajectory of the captured droplet evaluated from the recorded video frames over $1 \mathrm{~s}$ is depicted. It took several tens of seconds until the captured droplet settles into the stable position within $0.01-0.02 \mathrm{~mm}$ from such a few millimeters of the initial displacement.

The condition for the stable production of single droplets depends on the droplet radius, fluid type, as well as the exact geometry and the method of the droplet dispensers. ${ }^{20}$ In the case of water droplets with the surface tension $\sigma(=0.072 \mathrm{~N} / \mathrm{m})$ and $R$ in the range of $0.1-0.3 \mathrm{~mm}$, the ejection velocity $U_{e}$ should be set in the range of $0.5-1$ $\mathrm{m} / \mathrm{s}^{21}$ This is because, on one hand, the fluid should acquire a velocity $U_{l}$ to compensate the Laplace pressure $2 \sigma / R$ inside the droplet with $U_{e}$ being a factor 2-3 lower than $U_{l}{ }^{21,22}$ and on the other hand, $U_{e}$ should be limited within a certain value to avoid the splashing and the formation of satellite droplets with significant volumes. ${ }^{21,23}$ Because of the former requirement, decreasing $U_{e}$ to the value of $0.44 \mathrm{~m} / \mathrm{s}$ as in Fig. 2 or lower tends to make the ejection process unstable.

To circumvent the low- $U_{e}$ requirement and to shorten the settling time of the captured droplet, we used two DoD dispensers, Fig. 3(a), to eject two counter propagating droplets. The droplet loading was achieved upon coalescence $e^{24-30}$ at the pressure node. In this way, we were able to dispense droplets at a $U_{e}$ value of $0.5-1 \mathrm{~m} / \mathrm{s}$ in the stable dispenser operation. This also simplified the alignment procedure of the dispensers.
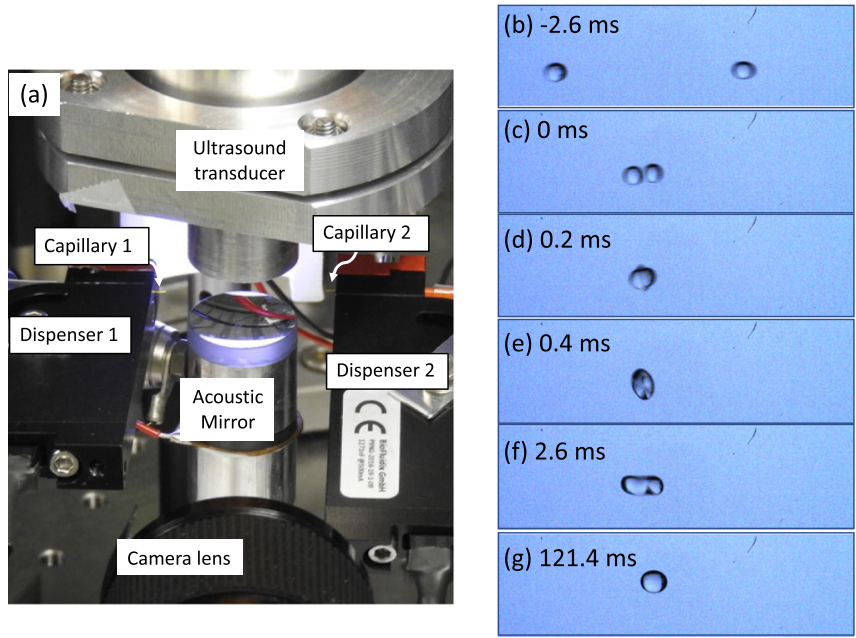

FIG. 3. (a) The experimental configuration of the droplet loading to the acoustic levitator using two drop-on-demand dispensers. (b)-(g) show the selected time frames of the droplet loading experiment recorded using the high-speed camera at the frame rate of $5000 \mathrm{~Hz}$. The droplet radii were equal to $0.26 \mathrm{~mm}$, and the relative velocity of the droplets was equal to $1.65 \mathrm{~m} / \mathrm{s}$.
In Figs. 3(b)-3(g), we show a recorded loading event of a water droplet with the radius $R_{l}$ of $\sim 0.33 \mathrm{~mm}$ as a result of the coalescence of two droplets with the radius $R_{d}$ of $0.26 \mathrm{~mm}$ (also see supplementary material Video 2). Instead of the 3rd resonance, the acoustic levitator was adjusted to the 5 th resonance with a $P_{a c}$ value of $3.2 \mathrm{kPa} \mathrm{rms}$. The relative velocity $U$ of the two droplets was equal to $1.65 \mathrm{~m} / \mathrm{s}$, with the individual droplet velocity equal to 0.75 and $0.90 \mathrm{~m} / \mathrm{s}$, for the left and right droplets, respectively. The stabilization time of the droplet position after the loading was an order of magnitude smaller in the present double-dispensing method than the single dispenser case because of the order of magnitude smaller initial amplitude, even though the time constant of the decay of the droplet oscillations was nominally the same in both cases. After the impact and the coalescence of two droplets, the droplet shape oscillated for $\sim 0.1 \mathrm{~s}$. This duration agrees with the time constant $\rho R_{l}^{2} / \eta$ (where $\eta=0.89 \mathrm{mPa}$ s is the dynamic viscosity of water at $25^{\circ} \mathrm{C}$ ) equal to $0.07 \mathrm{~s}$ calculated for the surface oscillation of spherical droplets. ${ }^{31}$ When the impact parameter (the distance between the droplet centers at the time of the droplet collision) was larger than the present case, the coalesced droplet exhibited a rapid rotation that decayed within $\sim 0.1 \mathrm{~s}$.

By varying the dispenser conditions, we found that we were able to load droplets with an $R_{l}$ value of $\sim 0.3 \mathrm{~mm}$ stably, when two droplets were ejected with $U$ in the range of $1.4-1.8 \mathrm{~m} / \mathrm{s}$ with the impact parameter being within $\sim 0.3 \mathrm{~mm}$. The corresponding Weber number given by $2 R_{l} \rho U^{2} / \sigma$ was in the range of 16.3-27.0. When $U$ was increased to $2.0 \mathrm{~m} / \mathrm{s}$ at the impact parameter of $\sim 0.3 \mathrm{~mm}$, the droplets disrupted upon impact. This agrees with the experiment reported by Adam et al. ${ }^{24}$ In the literature, coalescence events of droplets at Weber numbers higher than 80 were reported. However, such conditions demanded to set the impact parameter within a narrow range. ${ }^{24,28}$ Therefore, increasing $U$ larger than $2 \mathrm{~m} / \mathrm{s}$ is not practical from the application point of view. For robust coalescence of two dispensed droplets, we found that the individual droplet velocity should be larger than $\sim 0.7 \mathrm{~m} / \mathrm{s}$. Otherwise, the droplet trajectories bounced vertically, which had to be compensated by the dispenser alignment.

We repeated the droplet loading experiment using $0.2 \mathrm{~mm}$ capillaries (see supplementary material Video 3 ). In this case, we set $U_{e}$ in the range of $0.65-1.8 \mathrm{~m} / \mathrm{s}$ and $U$ in the range of $1.7-3.2 \mathrm{~m} / \mathrm{s}$. The $R_{d}$ values were equal to $\sim 0.15 \mathrm{~mm}$, and therefore, $R_{l}$ and the volume of the loaded droplet were equal to $0.20 \mathrm{~mm}$ and $31 \mathrm{nl}$, respectively. The impact parameter was kept within $\sim 0.1 \mathrm{~mm}$. Loading of the droplet without disruption upon impact was possible when $U$ was below $\sim 2.7 \mathrm{~m} / \mathrm{s}$ (the corresponding maximum Weber number equal to 40.5). Compared to the case of $R_{l}=0.3 \mathrm{~mm}$, this was 1.5 times larger as expected from the fact that the maximum Weber number increases approximately inversely proportional to $R_{l}{ }^{24,28}$ At the higher $U$, droplets disrupted upon impact. When the velocities of disrupted droplets were sufficiently low, those were reunited by the acoustic radiation force (see supplementary material Video 4). However, lower $U$ below $\sim 2.7 \mathrm{~m} / \mathrm{s}$ is more practical because of the tendency to generate satellite droplets at high $U_{e}$ values larger than 1.4-1.6 m/s. Supplementary material Video 5 shows five consecutive droplet loading events, when droplets were loaded into the levitator, demonstrating the stability of the double DoD dispenser method for the droplet loading.

$\mathrm{X}$-ray diffraction experiments of the lysozyme crystals loaded into the ALD by the double-dispenser method were performed to illustrate the properties of the method. After loading the droplet and 
confirming single crystals therein, the X-ray beam was exposed for $3 \mathrm{~s}$ to collect 3000 diffraction images at the frame rate of $1 \mathrm{kHz}$. As shown in the inset in Fig. 4(a), sharp diffraction spots could be recorded. This indicated the absence of crystal damage, that is, the present sample dispensing method and the data collection using the ultrasound acoustic levitation caused neither the increase in crystal mosaicity nor the changes in unit-cell parameters. ${ }^{32}$ The collected 9 datasets were analyzed by using the CrystFEL serial crystallography data analysis software $^{33}$ to identify the crystal orientations and the unit cell sizes and to assign reciprocal space indexes of the observed Bragg spots for each image independently (indexing). Using one of the datasets obtained from single crystals, we solved the crystal structure by the molecular replacement method using CCP $4 .{ }^{34}$ For this dataset, we found that the hit rate was 30\% (the fraction of images recorded the diffraction from the crystal) and the index rate was $10 \%$ (the fraction of images of which Bragg spot indexes were assigned). Other datasets were discarded because of the low index rate or because the droplet contained multiple crystals. The achieved resolution of the dataset was approximately equal to $2.65 \AA$ (for statistics, see supplementary material Table S1) based on $\mathrm{CC}_{1 / 2}$ of 0.067 for the 354 reflection pairs, where $\mathrm{CC}_{1 / 2}$ is Pearson's correlation function calculated between randomly split two halves of the data. ${ }^{35}$ According to the Student's t-test, ${ }^{36}$ this is significantly different from zero at $90.4 \%$ confidence. The resolution was limited in the present experiment due to the used X-ray wavelength (equal to $1.24 \AA$ ), the distance between the sample and the detector, and the detector area (the resolutions at the sides and the corners of the detector area were $2.9 \AA$ and $2.2 \AA$, respectively).

In Figs. 4(b) and 4(c), we show the electron density map of the solved lysozyme structure near the 20th to 25th amino acid residues and near the disulfide bridge between the 64th and 80th residues,
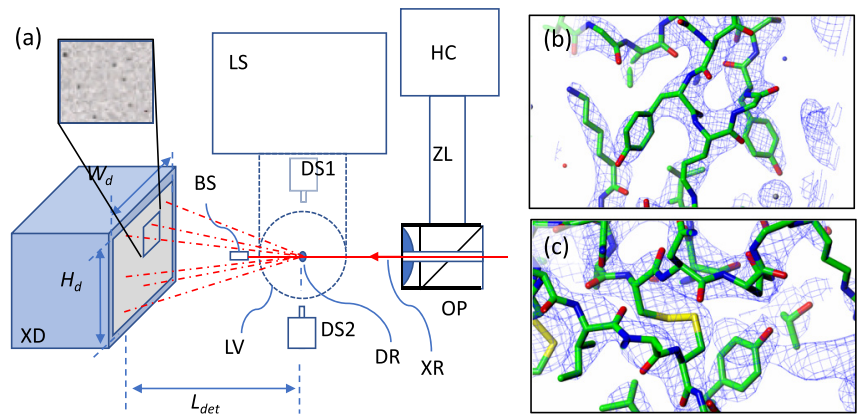

FIG. 4. (a) Schematic of the ALD experimental setup. The schematics in the middle show the top view of the acoustic levitator, drop-on-demand dispensers, and the camera optics. LV: acoustic levitator, DR: droplet, XR: X-ray beam, XD: fast-framerate $\mathrm{X}$-ray image detector, $\mathrm{OP}$ : objective lens-prism assembly, ZL: zoom lens, HC: high-speed camera for droplet observation, DS1 and 2: drop-on-demand dispensers, BS: beam stop for the direct X-ray beam, LS: support for the acoustic levitator with the $X Y Z$ translation stage, Ldet: distance between the sample in the levitating droplet and the detector equal to $85 \mathrm{~mm}$, and $\mathrm{Wd}$ : width of the $\mathrm{X}$-ray detector active area equal to $77.2 \mathrm{~mm}$ (the height was equal to $79.9 \mathrm{~mm}$ ). The inset shows the sharp diffraction spots observed in the experiment. (b) and (c) show the electron density maps of the solved lysozyme structure, rendered by CCP4MG: ${ }^{37}$ (b) near the 20th to 25th residues and (c) near the disulfide bridge (yellow skeleton) between the sulfur atoms at the 64th and 80 th residues. The space group of the crystal was $P 4_{3} 2_{1} 2$, and the unit cell parameters were equal to $a=78.82 \AA$ and $c=38.11 \AA$. These were consistent with the typical parameters of lysozyme crystals. respectively. These are shown at a resolution limit of $2.8 \AA$ at the contour level of $1.5 \sigma$, where $1 \sigma$ corresponds to the background noise level. The electron density map is superposed by the molecular skeleton, representing carbon by green, sulfur by yellow, nitrogen by blue, and oxygen by red. The bond lengths of the lysozyme structure obtained by the present experiment agree well with the previous results within $0.5 \%$. In particular, we found that the length of the disulfide bridge evaluated from Fig. 4(c) and that for 3 other disulfide bridges at other parts of the molecule (see supplementary material Table S2 and supplementary material Fig. S2) were the same as the values obtained in previous experiments reported in Ref. 4. Disulfide bridge lengths of refined structures have been used as a benchmark of X-ray radiation damage, partly because the S-S bonding energy is the weakest in typical protein molecules $(\sim 30 \%$ smaller than the bonding energy between two carbon atoms). Whether disulfide bridge lengths are also good indicators for the damage on the crystal by acoustic radiation is not known yet and needs further investigation. Nevertheless, together with the observed sharp diffraction spots, our result indicates the absence of the crystal damage by the drop-on-demand dispensing method. ${ }^{32}$ This conclusion is in line with the literature reporting the crystallography experiments for ejected crystals from drop-on-demand dispensers. ${ }^{11-15}$

In summary, we developed a method of on-demand droplet loading into an acoustic levitator using DoD dispensers and demonstrated a pilot crystallography experiment for single protein crystal samples loaded to ALD using this method. Combining the crystal harvesting and delivery steps in a single on-demand step together with the fast data collection using ALD, we consider that this is a significant advancement to realize a high throughput and fully automated crystallography pipeline. The prospective orders-of-magnitude improvement of the throughput will be highly beneficial for screening of large ligand libraries for drug discovery, ${ }^{1,38,39}$ which enables the fully experimental $\mathrm{X}$-ray screening of compound libraries consisting of several thousand of fragment molecules or compounds selected by structure based insilico methods. For a fully automated system without the requirement of manual work, it is highly desirable to avoid the step to fill the dispenser capillary with samples. The development of such a sample loading method, e.g., acoustic ejection of samples from standard crystallization plates, ${ }^{15}$ will be highly beneficial to improve the overall throughput further and to realize a fully automated crystallography pipeline utilizing ALD. As the method works at room temperature, crystal cryo-freezing is not required. This avoids the potential damage of the crystals during the cryo-freezing. Even more importantly, it gives X-ray structure information at ambient temperature closer to physiological conditions, showing structural flexibility and multiple conformations. $^{40-42}$

See the supplementary material for the schematic of the Schlieren imaging setup (Fig. S1), statistics of the crystallography data (Table S1), S-S distances (Table S2), other electron density maps (Fig. S2), droplet loading into the acoustic levitator using the single DoD dispenser (supplementary material Video 1) and the double DoD dispenser (supplementary material Video 2 with $500 \mu \mathrm{m}$ capillaries, supplementary material Video 3 with $200 \mu \mathrm{m}$ capillaries, and supplementary material Video 4 with $200 \mu \mathrm{m}$ capillaries at high ejection velocities), and repeated droplet loading using double $\mathrm{DoD}$ dispensers using $200 \mu \mathrm{m}$ capillaries (supplementary material Video 5). 
The authors acknowledge A. Shirahama for preparing the protein crystal samples, M. Henning for enlightening discussions on the demands for drug discovery and critical reading of the manuscript, and E. Rüfenacht for her help to study the ejector performances. This work was partially supported by Innosuisse in a Project No.18726.1 in collaboration with leadXpro AG.

\section{REFERENCES}

${ }^{1}$ G. M. Keserue, D. A. Erlanson, G. G. Ferenczy, M. M. Hann, C. W. Murray, and S. D. Pickett, J. Med. Chem. 59, 8189 (2016).

${ }^{2}$ F. U. Huschmann, J. Linnik, K. Sparta, M. Uhlein, X. Wang, A. Metz, J. Schiebel, A. Heine, G. Klebe, M. S. Weissa, and U. Muellera, Acta Crystallogr., Sect. F 72, 346 (2016).

${ }^{3}$ P. McEwan, P. Collins, A. Dias, M. Mazanetz, B. Brandao-Neto, A. Douangamath, J. Ng, N. Wright, T. Krojer, T. R, J. Barker, and F. von Delft, in Proceedings of Fragments 2015: Fifth RSC-BMCS Fragment-Based Drug Discovery Meeting, 2015.

${ }^{4}$ S. Tsujino and T. Tomizaki, Sci. Rep. 6, 25558 (2016).

${ }^{5}$ E. Welter and B. Neidhart, Fresenius' J. Anal. Chem. 357, 345 (1997).

${ }^{6}$ Y. Abe, D. Hyuga, S. Yamada, and K. Aoki, Ann. N. Y. Acad. Sci. 1077, 49 (2006).

${ }^{7}$ K. Kobayashi, A. Goda, K. Hasegawa, and Y. Abe, Phys. Fluids 30, 082105 (2018).

${ }^{8}$ E. G. Lierke, Acustica 82, 220 (1996).

${ }^{9}$ J. H. Hubbel and S. M. Seltzer, "Tables of x-ray mass attenuation coefficients and mass energy-absorption coefficients from $1 \mathrm{kev}$ to $20 \mathrm{mev}$ for elements $\mathrm{z}=1$ to 92 and 48 additional substances of dosimetric interest," NIST Standard Reference Database 126 (2004).

${ }^{10} \mathrm{~T}$. Earnest and C. Cork, International Tables for Crystallography, edited by E. Arnold, D. M. Himmel, and M. G. Rossmann (Wiley-VCH, Weinheim 2012), Vol. F.

${ }^{11}$ A. S. Soares, M. A. Engel, R. Stearns, S. Datwani, J. Olechno, R. Ellson, J. M. Skinner, M. Allaire, and A. M. Orville, Biochemistry 50, 4399 (2011).

${ }^{12}$ C. G. Roessler, R. Agarwal, M. Allaire, R. Alonso-Mori, B. Andi, J. F. Bachega, M. Bommer, A. S. Brewster, M. C. Browne, R. Chatterjee, E. Cho, A. E. Cohen, M. Cowan, S. Datwani, V. L. Davidson, J. Defever, B. Eaton, R. Ellson, Y. Feng, L. P. Ghislain, J. M. Glownia, G. Han, J. Hattne, J. Hellmich, A. Heroux, M. Ibrahim, J. Kern, A. Kuczewski, H. T. Lemke, L. M. P. Liu, W. M. McClintock, S. Myers, S. Nelsen, J. Olechno, A. M. Orville, N. K. Sauter, A. S. Soares, S. M. Soltis, H. Song, R. G. Stearns, R. Tran, Y. Tsai, and M. Uervirojnangkoorn, Structure 24, 631 (2016).

${ }^{13}$ F. Mafune, K. Miyajima, K. Tono, Y. Takeda, J. Kohno, N. Miyauchi, Y. Joti, J. Kobayashi, E. Nango, S. Iwata, and M. Yabashi, Acta Crystallogr., Sect. D 72, 520 (2016).

${ }^{14}$ F. D. Fuller, S. Gul, R. Chatterjee, E. S. Burgie, I. D. Young, H. Lebrette, V. Srinivas, A. S. Brewster, T. Michels-Clark, J. A. Clinger, B. Andi, M. Ibrahim, E. Pastor, C. de Lichtenberg, R. Hussein, C. J. Pollock, M. Zhang, C. A. Stan, T. Kroll, T. Fransson, C. Weninger, M. Kubin, P. Aller, L. Lassalle, P. Bräuer, M. D. Miller, M. Amin, S. Koroidov, C. G. Roessler, M. Allaire, R. G. Sierra, P. T. Docker, J. M. Glownia, S. Nelson, J. E. Koglin, D. Zhu, M. Chollet, S. Song, H. Lemke, M. Liang, D. Sokaras, R. Alonso-Mori, A. Zouni, J. Messinger,
U. Bergmann, A. K. Boal, J. M. Bollinger, Jr., C. Krebs, M. Hogbom, G. N. Phillips, Jr., R. D. Vierstra, N. K. Sauter, A. M. Orville, J. Kern, V. K. Yachandra, and J. Yano, Nat. Methods 14, 443 (2017).

${ }^{15}$ Y. N. Samara, H. M. Brennan, L. McCarthy, M. T. Bollard, D. Laspina, J. M. Wlodek, S. L. Campos, R. Natarajan, K. Gofron, S. McSweeney, A. S. Soares, and L. Leroy, Acta Crystallogr., Sect. D 74, 986 (2018).

${ }^{16}$ G. S. Settles, Schlieren Shadow Graph Techniques: Visualizing Phenomena in Transparent Media (Springer Verlag, Berlin, Heidelberg, 2001), p. 25.

${ }^{17}$ S. Tsujino, Y. Sato, Y. Takeda, and T. Tomizaki (unpublished 2019).

${ }^{18}$ W. Streule, T. Lindemann, G. Birkle, R. Zengerle, and P. Koltay, JALA October, 300 (2004).

${ }^{19}$ T. Tomizaki, A. Shinoda, and S. Tsujino, AIP Conf. Ser. 2054, 060072 (2019).

${ }^{20}$ I. M. Hutchings, Inkjet Technology for Digital Fabrication (John Wiley \& Sons Ltd., 2013).

${ }^{21}$ S. A. Elrod, B. Hadimioglu, B. T. Khuri-Yakub, E. G. Rawson, E. Richley, C. F. Quate, N. N. Mansour, and T. S. Lundgren, J. Appl. Phys. 65, 3441 (1989).

${ }^{22}$ H. Tanaka, Y. Mizuno, and K. Nakamura, Jpn. J. Appl. Phys. Part I 56, 087202 (2017).

${ }^{23} \mathrm{~B}$. Derby, "Inkjet printing of functional and structural materials: Fluid property requirements, feature stability, and resolution," Annu. Rev. Mater. Res. 40, 395 (2010).

${ }^{24}$ J. R. Adam, N. R. Lindblad, and C. D. Hendricks, J. Appl. Phys. 39, 5173 (1968).

${ }^{25}$ P. R. Brazier-Smith, S. G. Jennins, and J. Latham, Proc. R. Soc. London, Ser. A. 326, 393 (1972).

${ }^{26}$ Y. J. Jiang, A. Umemura, and C. K. Law, J. Fluid Mech. 234, 171 (1992).

27.J. Qian and C. K. Law, J. Fluid Mech. 331, 59 (1997).

${ }^{28}$ M. Orme, Prog. Energy Combust. Sci. 23, 65 (1997).

${ }^{29}$ C.-Y. Lee, H. Yu, and E. S. Kim, Appl. Phys. Lett. 89, 223902 (2006).

30 J. Kohno, M. Kobayashi, and T. Suzuki, Chem. Phys. Lett. 578, 15 (2013).

${ }^{31}$ H. Lamb, Hydrodynamics, 6th ed. (Dover Publications, Inc., New York, 1932), p. 640.

${ }^{32}$ K. Diederichs, Acta Crystallogr., Sect. D 65, 535 (2009).

${ }^{33}$ T. A. White, R. A. Kirian, A. V. Martin, A. Aquila, K. Nass, A. Barty, and H. N. Chapman, J. Appl. Crystallogr. 45, 335 (2012).

${ }^{34}$ M. D. Winn, C. C. Ballard, K. D. Cowtan, E. J. Dodson, P. Emsley, P. R. Evans, R. M. Keegan, E. B. Krissinel, A. G. Leslie, A. McCoy, S. J. McNicholas, G. N. Murshudov, N. S. Pannu, E. A. Potterton, H. R. Powell, R. J. Read, A. Vagin, and K. S. Wilson, Acta. Crystallogr., Sect. D 67, 235 (2011).

${ }^{35} \mathrm{P}$. A. Karplus and K. Diederichs, Science 336, 1030 (2012).

${ }^{36}$ N. A. Rahman, A Course in Theoretical Statistics (Griffin, London, 1968), p. 456.

${ }^{37}$ S. McNicholas, E. Potterton, K. S. Wilson, and M. E. M. Noble, Acta Crystallogr., Sect. D 67, 386 (2011).

${ }^{38}$ J. M. Grimes, D. R. Hall, A. W. Ashton, G. Evans, R. L. Owen, A. Wagner, K. E. McAuley, F. von Delft, A. M. Orville, T. Sorensen, M. A. Walsh, H. M. Ginna, and D. I. Stuart, Acta Crystallogr., Sect. D 74, 152 (2018).

${ }^{39}$ P. Rucktooa, R. K. Y. Cheng, E. Segala, T. Geng, J. C. Errey, G. A. Brown, R. M. Cooke, F. H. Marshall, and A. S. Doré, Sci. Rep. 8, 41 (2018).

${ }^{40}$ S. Fraser, H. van den Badem, A. J. Samelson, P. T. Lang, J. M. Holton, N. Echos, and T. Alber, Proc. Natl. Acad. Sci. U. S. A. 108, 16247 (2011).

${ }^{41}$ M. Fischer, B. K. Shoichet, and J. S. Fraser, ChemBioCherm 16, 1560 (2015).

${ }^{42}$ R. K. Y. Chen, R. Abela, and M. Henning, Essays Biochem. 61, 529 (2017). 